\title{
Interfacing and Analysis of GPS data using VB
}

\author{
Rohit Dhull ${ }^{1}$, Prof. Sugandha Singh Hooda ${ }^{2}$ \\ ${ }^{1}$ Research Scholars CE Department, PDM College of Engineering, Bahadurgarh, India \\ ${ }^{2}$ Head, CSE Department, PDM College of Engineering, Bahadurgarh, India
}

\begin{abstract}
Global Positioning System (GPS) provides location and time information anywhere on the earth. We can utilize GPS data to create new applications that can be very useful nowadays. If we want to build a new application or system with GPS technology then we must analyse the GPS data and understand it systematically. The objective of this study is to interface the GPS module and analyse the GPS data. This paper presents a simple way to interface the GPS Module using VB.NET.
\end{abstract}

Keywords: Global Positioning System, Designated L1 signal, NMEA data, COM interoperability.

\section{Introduction}

Global Positioning System (GPS)is a satellite-based navigation system that provides exact location and time information on the earth. ${ }^{[5]}$ These satellites revolve around the earth twice a day in a precise orbit. GPS is a network of 31 satellites orbiting the earth at an altitude of $20,180 \mathrm{~km} .{ }^{[5]}$ The GPS satellite sends the data to the GPS receiver then GPS receiver process the data and get the precise location and time information. ${ }^{[3]}$ When the GPS receiver gets the signal from at least 3 satellites then it can calculate 2-D position (latitude and longitude) and with at least 4 satellites, the receiver can calculate the 3-D position (latitude, longitude and altitude). ${ }^{[1]}$ Nowadays we can get up to 3 meters accuracy with better GPS receivers. All the GPS satellites are solar powered and they have batteries that can power them at the time of solar eclipse or when there is no solar power. GPS satellites transmit radio signals, designated L1 and L2. Designated L1 radio signal can be accessed by common people at frequency $1575.42 \mathrm{MHz}$. Designated L1 can travel through clouds andplastic materialbut cannot get travel through solid objects like buildings, mountains etc. GPS signal contains two main information. ${ }^{[4]}$ First one is pseudorandom code, this code is a sequence of ones and zeros that is only known to the GPS receiver and second one is a message that consist of time of transmission and the position of the GPS satellite at that moment. ${ }^{[5],[2]}$ The working of Global Positioning System is shown in Figure 1.

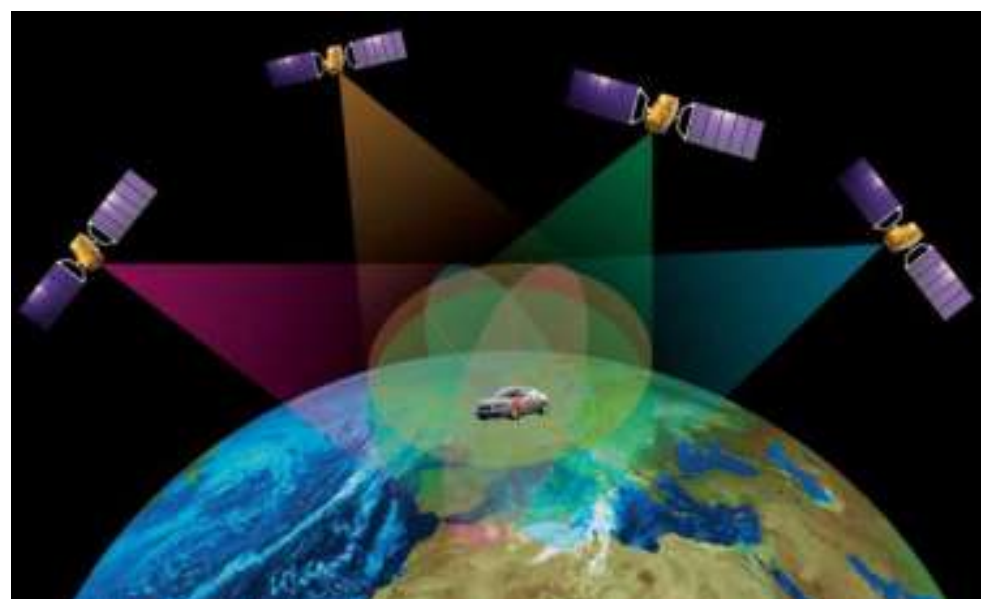

Figure 1: How Global Positioning System works

\section{GPS Module}

GPS Module that we have used is built with MT3329 chipset from MediaTek Inc. This module comes with rectangular micro strip antenna (also known as patch antenna). The tracking sensitivity of On-module antenna is $-165 \mathrm{dBm}$. We can also connect an external antenna to get better reception. The module automatically detects an external antenna and uses it with no user intervention required. It also has USB and Serial TTL Interface using which we can connect computer system to the GPS module and USB usesCP2102 IC to communicate with the system. We need to install CP210X Virtual COM Port (VCP) Drivers to make the communication possible between the module and the computer system. ${ }^{[1]}$ The GPS Module can be powered through USB or External power. The board can accept an external voltage between 3.3V - 5V supply. ${ }^{[14], ~[15] ~}$ 
The GPS module that we have used in this study is shown in Figure 2.

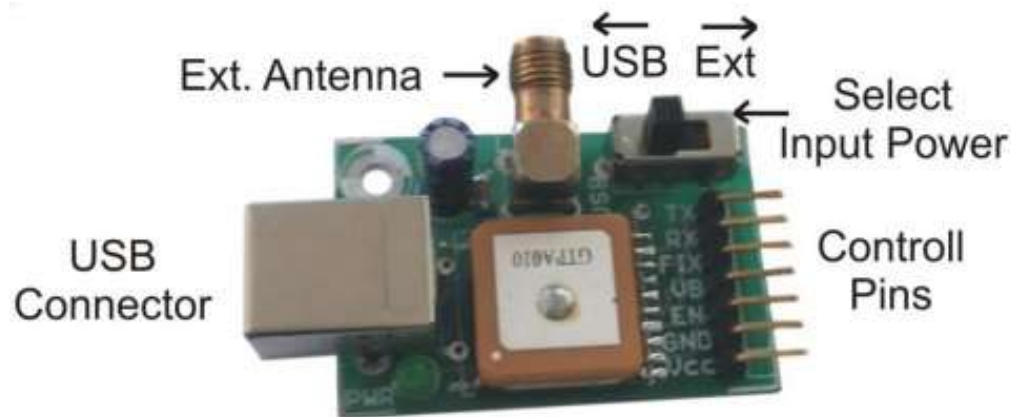

Figure 2: GPS Module

\section{GPS Module Interfacing}

When we connect the module to the computer system for the first time after the installation of drivers then it will create a new COM port. We can use that COM port to interface the module. After the COM port is created then the module will continuously output the GPS data to this port. Most of the programming languages allow users to write programs that can access the COM port. We have used VB.NET to create a program that can access the GPS data that we are receiving from the COM port. TheNational Marine Electronics Association (NMEA) has developed a standard that interpret the interaction between various electronic equipment. ${ }^{[7],[8]}$ TheGPS receiver follows these standards and receives NMEA data from the GPS satellites. NMEA data is a line of data that is called sentence and it is self-contained. ${ }^{[5],[6]}$

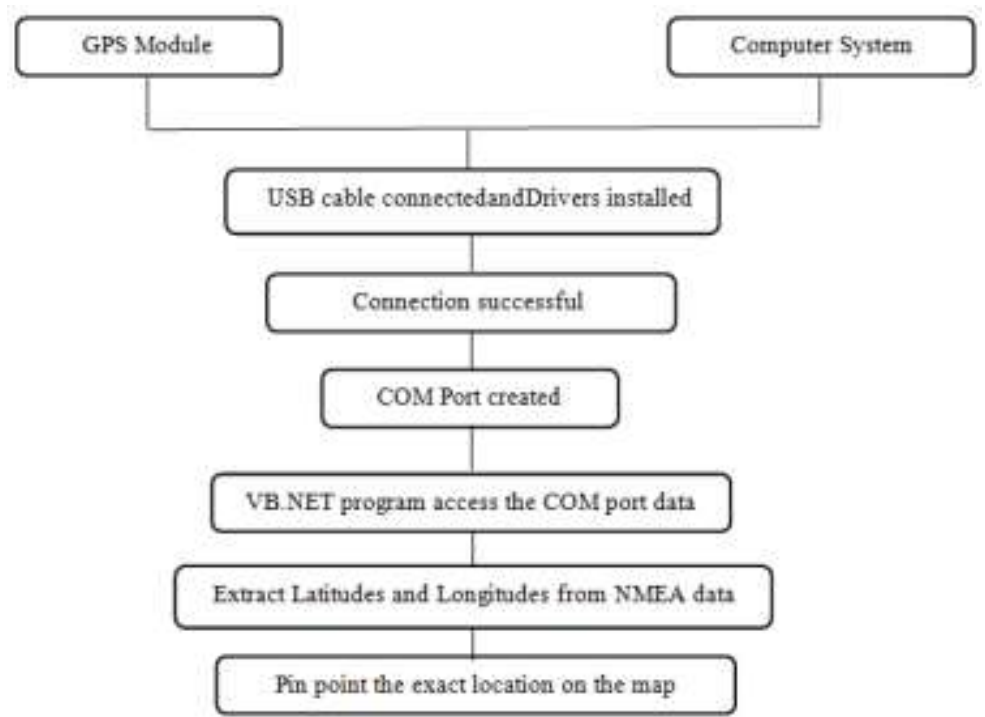

Block diagram of GPS module and computer system interfacing

All the data is separated with commas in the sentence. Each sentence starts with '\$GPGGA', GP means Global Positioning and GGA defines the current fixed information which provides 3-D location and precise data. ${ }^{[6]}$ Let us take an example of NMEA data to understand it in a better way.

NMEA Data: \$Gpgga,112015,3212.016,N,01341.000,E,1,08,0.7,453.2,M,37.6,M,, ${ }^{* 52}$

\begin{tabular}{|ll|l|}
\hline Data & Meaning \\
\hline a) & 112015 & Fixed location taken at 11:20:15 UTC \\
\hline b) & $3212.016, \mathrm{~N}$ & Latitude 32 degrees 12.016 minutes North \\
\hline c) & $01341.000, \mathrm{E}$ & Longitude 13 degrees 41.000 minutes East \\
\hline d) & 1 & GPS fix \\
\hline e) & 08 & Number of satellites tracking the location \\
\hline f) & 0.7 & Horizontal dilution of location \\
\hline g) & $453.2, \mathrm{M}$ & Altitude, Meters \\
\hline h) & $37.6, \mathrm{M}$ & Height of geoid \\
\hline i) & Empty field & Time in seconds since last update \\
\hline j) & Empty field & Station ID number \\
\hline k) & $* 52$ & Checksum data \\
\hline
\end{tabular}


We have built a program in VB.NET to interface the GPS module. VB.NET is an object oriented programming language that provide us many features like Error handling, COM interoperability, Simplified deployment of applications, Flexible etc. We can create powerful Windows based applications using VB.NET very easily. ${ }^{[13],[12]}$

Now we have the NMEA data as output of the GPS module but we don't need all the NMEA data to pin point the exact location, we just need Latitudes, Longitudes and directions. So we have built the program like this so that when we start the program it checks for the serial port whether it is open or not and if it is open then the program reads the output of the device into a string variable. Now we have to break the string array to get the desired data and then convert the Latitudes and Longitudes into decimal degrees. [9], [10]

The user interface of the program shows the Latitudes and Longitudes before and after the conversion to decimal degree and if we get the valid coordinates then we can get the precise location on the google map by clicking on the action button. But when the coordinates are invalid, the program will display message "GPS Unavailable" in the text boxes of latitude and longitude and if the serial port is closed or not functional then the these text boxes will be display the message "COM Port Closed" in either case, the action button will be disabled. We can only track our location when we get the valid coordinates. ${ }^{[13],[11]}$

Suppose we get the valid coordinates then after clicking on the mapping button, the program will open the web browser that is within the program then navigate it to the google maps website and automatically pass the latitudes and longitudes, this is how we will get our precise location. ${ }^{[13]}$

\section{Conclusion}

The program we have developed in VB.NET is a miniature of what we can do with GPS technology. We can use the GPS technology to create far better windows based and web based applications. Since cost being a constraint for further advancement, we can use simple GPS receiver with patch antenna to access the GPS data and use that with any project we want. We can develop user friendly and cost effective applications like tourist guide, vehicle tracking, etc. for the users.

\section{References}

[1]. J.Parthasarathy. "Positioning And Navigation System Using Gps," International Archives Of The Photogrammetry, Remote Sensing And Spatial Information Science, Volume Xxxvi, Part 6, Tokyo Japan 2006.

[2]. Pankaj Verma, J.S Bhatia. "Design And Development Of Gps-Gsm Based Tracking System With Google Map Based Monitoring," International Journal Of Computer Science, Engineering And Applications (Ijcsea), Vol.3, No.3, June 2013.

[3]. Shital Mohol, Amit Pavanikar, Ganesh Dhage. "Gps Vehicle Tracking System," International Journal Of Emerging Engineering Research And Technology, Volume 2, Issue 7, October 2014.

[4]. Ms. Bhalerao Pournima, Prof. V. B. Baru. "Gps Tracking And Controller For Car Black Box On Fpga," International Journal Of Advanced Research In Computer Science And Software Engineering, Volume 3, Issue 12, December 2013.

[5]. Lawrence Harte, Ben Levitan. "Global Positioning System,"2nd Edition, 2009.

[6]. Http://Aprs.Gids.N1/Nmea/ "Gps - Nmea Sentence Information".

[7]. Https://En.Wikipedia.Org/Wiki/Nmea_0183 "Nmea 0183".

[8]. Http://Gpsworld.Com/What-Exactly-Is-Gps-Nmea-Data/ "What Is Exactly Gps Nmea Data".

[9]. Http://Www.Tutorialspoint.Com/Vb.Net/Vb.Net_Strings.Htm "Vb.Net Strings".

[10]. Http://Vb.Net-Informations.Com/"Vb.Net Tutorial And Source Code".

[11]. Http://Www.Codeproject.Com/Kb/Vb/"Vb.Net".

[12]. Jesse Liberty, Dan Hurwitz, "Programming .Net Windows Applications", October 2003.

[13]. Charles Petzold, "Programming Microsoft Windows With Microsoft Visual Basic .Net", 31 Aug 2002.

[14]. Http://Www.Circuitsgallery.Com/2015/11/Gps-Module-Configuration-Usb-Interface.Html "Gps Module Configuration Using UsbInterface And Sirfdemo".

[15]. Https://Www.Circuitsathome.Com/Mcu/Communicating-To-Gps-Receiver-Using-Usb-Host-Shield “Communicating To Gps Receiver Using Usb Host Shield". 